Research Article

\title{
Intelligent Extremum Surrogate Modeling Framework for Dynamic Probabilistic Analysis of Complex Mechanism
}

\author{
Jia-Qi Liu $\mathbb{D}$, Yun-Wen Feng $\mathbb{D}$, Xiao-Feng Xue, and Cheng Lu $\mathbb{D}$ \\ School of Aeronautics, Northwestern Polytechnical University, Xi'an 710072, China \\ Correspondence should be addressed to Yun-Wen Feng; fengyunwen@nwpu.edu.cn
}

Received 22 December 2020; Revised 4 January 2021; Accepted 1 February 2021; Published 15 February 2021

Academic Editor: Zuowei Cai

Copyright ( $\odot 2021$ Jia-Qi Liu et al. This is an open access article distributed under the Creative Commons Attribution License, which permits unrestricted use, distribution, and reproduction in any medium, provided the original work is properly cited.

\begin{abstract}
The reliability analysis of complex mechanisms involves time-varying, high-nonlinearity, and multiparameters. The traditional way is to employ Monte Carlo (MC) simulation to achieve the reliability level, but this method consumes too much computing resources and is even computationally intractable. To improve the efficiency and accuracy of dynamic probabilistic analysis of complex mechanisms, an intelligent extremum surrogate modeling framework (IESMF, short for) is proposed based on extremum response surface method (ERSM), combined with artificial neural network (ANN) method and an improved optimize particle swarm optimization (PSO) method. Hereinto, the ERSM is used to simplify the dynamic process of output response to the extremum value of transient analysis; ANN is applied to establish a mathematical model between input variables and response, and the improved PSO method is utilized in search of initial weights and thresholds of the model. The effectiveness of the IESMF is demonstrated to perform the Rack-and-pinion steering mechanism (RPSM) reliability analysis. The results show that when the allowable value of gear root stress is equal to $850 \mathrm{MPa}$, the RPSM has a reliability degree of 0.9971 . Through the validation process, it is illustrated that IESMF is accurate and efficient in dynamic probabilistic analysis of complex mechanisms, and its comprehensive performance is better than the MC method and ERSM. The research effort offers new ideas for the reliability estimation of a complex mechanism, thus enriching the method and theory of mechanical reliability design.
\end{abstract}

\section{Introduction}

For complex mechanisms, e.g., the steering mechanism of the aircraft nose wheel, its reliability level is seriously affected by many time-varying factors, such as speed, acceleration, load, and so on [1-3]. The limit state equation of complex mechanisms in probabilistic analysis has the characteristics of being highly nonlinear and multivariable. This leads to some problems in the process of reliability analysis, a large amount of calculation, and difficulty in ensuring the accuracy of calculation, which leads to the difficulty of mechanism reliability analysis [4-6]. Therefore, to improve the performance of the mechanical system, the randomness of input parameters must be considered in mechanism reliability analysis. Some scholars have carried out many numerical and experimental studies on complex mechanisms through deterministic analysis methods [7-9]. However, these studies have not paid enough attention to various uncertain factors affecting the performance of complex mechanisms. The probabilistic analysis involves random factors such as material parameters and physical field loads. It has acceptable accuracy in describing the failure response and is a feasible alternative method [10-12].

As an important technology, the application of probability analysis in many fields has been widely studied [13-15]. Nonetheless, the application of probabilistic analysis in the reliability research of complex mechanisms with multiple influencing factors is rare. When performing probabilistic analysis, traditional methods include direct simulation methods such as Monte Carlo (MC) [16-18] and surrogate model methods such as response surface method (RSM) [19-21]. In recent years, extreme response surface methods (ERSM) have also been developed [22-24]. Because of its high accuracy in reliability design and evaluation, MC simulation has been favored by researchers in many fields in recent years, whereas MCM cannot meet the requirements of solving highnonlinearity limit state functions. As one of the key points of probabilistic analysis of complex mechanism, RSM can 
improve computational accuracy and efficiency. However, RSM needs to establish a great number of objective functions in the process of mechanism dynamic probability analysis, which may lead to unacceptable calculation. To deal with the high-nonlinearity and time-varying problems in the reliability estimation of complex mechanisms, ERSM does not care about the output response of the mechanism other than extreme values. However, the fitting accuracy and efficiency of extremum response surface function in the probabilistic analysis are not sufficient, so it is difficult to describe the dynamic characteristics and strong nonlinearity in dynamic probabilistic analysis of complex mechanisms. Therefore, we need to develop an efficient and accurate model by improving ERSM to further strengthen the probabilistic analysis capabilities of complex mechanisms.

To improve the approximation ability and computational efficiency of ERSM, a feasible method is to establish a high-precision extremum response surface function based on the extreme value surrogate model. Artificial neural network (ANN) is an intelligent learning method with excellent robustness and nonlinear mapping ability. ANN performs well in the application of pattern recognition and data mining due to its advantages of fewer training samples, high computational efficiency, and high-precision [25-28]. According to the excellent characteristics of ANN, we propose a dynamic probability analysis method (ANNERSM), which combines ERSM and ANN. The outstanding simplified computing ability of ERSM and the powerful nonlinear mapping function of ANN are integrated into this method. Nevertheless, in the process of fitting high-nonlinearity, multiparameters, and time-varying limit state functions, there are always local optimization and overfitting problems in the training process, which affect the prediction accuracy, and its further application in the probability design of complex mechanisms is limited.

The object of this study is to propose an intelligent extremum surrogate modeling framework (IESMF) based on ERSM, combined with an ANN model and an improved optimize particle swarm optimization (PSO) method. The improved PSO is used to search the initial thresholds and weights of the model. The feasibility and effectiveness of IESMF are analysed by the rack-and-pinion steering mechanism of the nose landing gear.

In what follows, the basic theory of IESMF combined with ERSM, ANN, and improved PSO for probabilistic analysis of complex mechanisms is discussed in Section 2. We describe the dynamic probabilistic analysis program using IESMF in Section 3. In Section 4, the proposed method is verified by the reliability analysis of the rack-and-pinion mechanism of the nose wheel steering system. We close the paper with some conclusions in Section 5.

\section{Basic Theory}

2.1. Basic Thought of ERSM. ERSM is developed by applying the extremum theory to the traditional RSM to overcome the shortcoming that a large number of objective functions need to be established in the process of probability analysis of mechanisms with time-varying characteristics. It simplifies the analysis process, improves the calculation efficiency, and provides a new way for probabilistic analysis. The basic idea of dynamic probability analysis of mechanism based on ERSM is [23] using the extreme value idea to determine the extreme value of the dynamic output response of the mechanism, obtain the sample of variable parameters, and establish the objective output function; combining the numerical simulation method or approximate analytical theory to realize the dynamic reliability analysis of mechanism. We use the idea of extreme value to deal with the dynamic process of output response and simplify it to transient analysis with extreme values. The mathematical model of the objective output function is constructed to replace the real simulation, which can effectively improve the calculation efficiency of dynamic probability analysis [24]. Figure 1 shows the basic principle of ERSM.

Assuming that $\mathbf{Y}_{j}\left(t, \mathbf{x}_{j}\right)(j=1,2, \ldots, m)$ is the output response of the $j$ th input vector $\mathbf{x}_{j}, \mathbf{Y}_{j \text {, max }}\left(\mathbf{x}_{j}\right)$ is the dynamic extremum of $\mathbf{Y}_{j}\left(t, \mathbf{x}_{j}\right)$ in the time domain $[0, T]$. The extreme response surface function $f(\mathbf{x})$ is fitted by the data set $\left\{\mathbf{Y}_{j \text {, max }}\right.$ $\left(\mathbf{x}_{j}\right): j=1,2, \ldots, m, m$ is the number of samples $\}$ consisting of the maximum output response and the extreme response curve $\mathbf{Y}(\mathbf{x})$ is donated by the following:

$$
Y(\mathbf{x})=f(\mathbf{x})=\left\{Y_{j, \max }\left(\mathbf{x}_{j}\right)\right\} .
$$

For ERSM, its function is usually described by quadratic polynomials. The above equation can be written as follows:

$$
Y_{\mathrm{ERSM}}(\mathbf{x})=a+\mathbf{b x}+\mathbf{x}^{\mathrm{T}} \mathbf{c x},
$$

where $a$ is the constant coefficient; $\mathbf{b}$ is the vector of a linear term; $c$ is the matrix of the quadratic term. Among them, $\mathbf{b}$ and $\mathbf{c}$ can be expressed as follows:

$$
\begin{aligned}
& \mathbf{b}=\left(\begin{array}{llll}
b_{1} & b_{2} & \ldots & b_{n}
\end{array}\right), \\
& \mathbf{c}=\left(\begin{array}{cccc} 
& & c_{11} & \\
& & & \\
& c_{21} & c_{22} & \\
& \vdots & \vdots & \vdots \\
& & & \\
c_{n 1} & c_{n 2} & \ldots & c_{n n}
\end{array}\right) .
\end{aligned}
$$

Based on Equations (3) and (4), the ERSM function is written as follows:

$$
Y_{\mathrm{ERSM}}(\mathbf{x})=a+\sum_{i=1}^{n} b_{i} x_{i}+\sum_{i=1}^{n} \sum_{i=1}^{n} c_{i \bar{i}} x_{i} x_{\bar{i}},
$$

where $\bar{i}=1,2, \ldots, n, n$ is the number of random variables.

Although ERSM can effectively improve the calculation efficiency of dynamic probability analysis of mechanism, its analysis accuracy cannot meet the engineering requirements. The main reason is that the modeling accuracy of traditional ERSM is limited by the quadratic polynomial itself, which cannot effectively reflect the relationship between input variables and output response for highly nonlinear problems; in addition, it is unable to reasonably determine and use effective sample data for modeling, which makes the predicted value deviate from the real simulation 


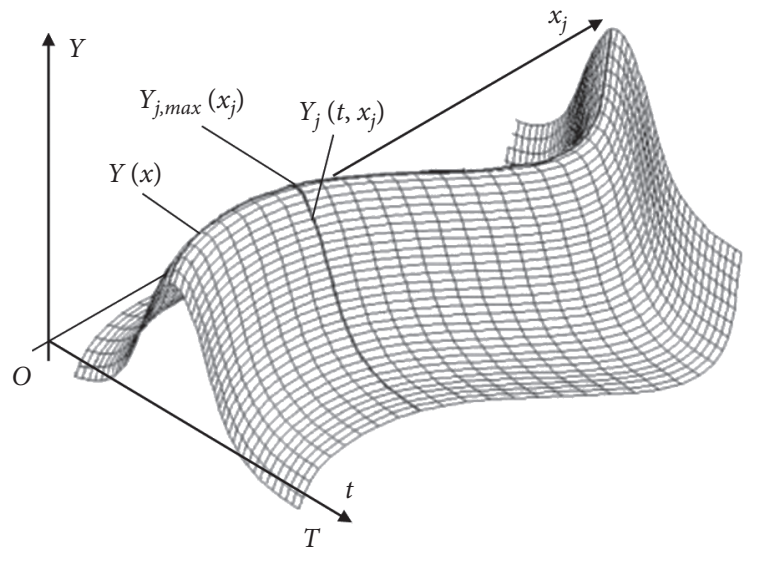

Figure 1: Basic thought of ERSM.

results. Extremum response surface function is an important part of dynamic probabilistic analysis. Effective extremum response surface function can improve efficiency and accuracy. To solve this problem, we need to establish an efficient and high-accuracy fitting model.

2.2. ERSM Based on ANN, ANN-ERSM. To reasonably use the sample data to establish a high-precision model meeting the engineering requirements, the back-propagation artificial neural network (BP-ANN) model is selected to fit the extremum response surface function of the limit state equation, namely ANN-ERSM. This method takes advantage of the nonlinear mapping of ANN and fits the high-nonlinearity extreme response surface to replace the dynamic equation, which improves the computational accuracy and efficiency. The artificial neural network has strong adaptability, its shape is flexible and changeable, and it can be more accurate in fitting the complex functional relationship between random variables and output response [29]. Figure 2 shows the BP-ANN topology model.

According to the idea of ERSM, the ANN regression function $f(\boldsymbol{x})$ is used to fit the input random variables $\mathbf{x}$ $\left(\mathbf{x} \in \mathbf{R}^{n}\right)$ and the dynamic extremum response $\mathbf{Y}_{j, \max }\left(\mathbf{x}_{\mathbf{j}}\right)$ $(\mathbf{y} \in \mathbf{R})$. The ANN-ERSM function is donated by the following:

$$
f(\mathbf{x})=\left\{Y_{j, \max }\left(\mathbf{x}_{j}\right)=f(\mathbf{x}, \mathbf{W}), \mathbf{W} \in \Lambda \mid f: \mathbf{R}^{n} \longrightarrow \mathbf{R}\right\},
$$

where $\mathbf{W}$ is the weight and threshold vector of ANN-ERSM, respectively.

Through the above analysis, we transformed the fitting problem of ANN-ERSM into a search problem of the optimal weight and threshold of the neural network. Combined with the idea of three-layer BP-ANN, the training performance function of ANN-ERSM is represented by the following:

$$
y=f_{2}\left(\sum_{i=1}^{n} \mathbf{W}_{\mathrm{ij}} f_{1}\left(\sum_{j=1}^{m} \mathbf{W}_{j k} \mathbf{x}_{i}+\mathbf{b}_{j}\right)+\mathbf{b}_{k}\right),
$$

where $\mathbf{b}_{j}$ is the $j$ th threshold value of hidden layer; $\mathbf{W}_{i j}$, the connection weight between the $i$ th node of the input layer and the $j$ th node of a hidden layer; $\mathbf{b}_{k}$, the kth threshold value of the output layer; $\mathbf{W}_{j k}$, the connection weight between the $j$ th node of hidden layer and the $k_{\text {th }}$ node of output layer; $f_{1}$ $(\cdot)$ is the transfer function of a hidden layer; $f_{2}(\cdot)$ is the transfer function of output layer; $m$ is the number of nodes in a hidden layer; $n$ is the number of nodes in the input layer.

To prevent the training algorithm from falling into the local optimum and immature convergence, this research uses the Bayesian regularization (BR) algorithm for network training, which has strong generalization ability. By adding prior conditions, the solution space is reduced and the possibility of finding wrong solutions is reduced. Studies have shown that smaller weights and thresholds can effectively reduce the occurrence of overfitting and have faster convergence characteristics [11]. We reduce the network weights to reduce training errors and improve training capability, avoid overfitting of BP-ANN, and improve calculation accuracy. The training performance function $\boldsymbol{E}$ based on BR is expressed as follows:

$$
\mathbf{E}=k_{1} \mathbf{E}_{D}+k_{2} \mathbf{E}_{W}
$$

where $k_{1}$ and $k_{2}$ are the proportional coefficients; $\mathbf{E}_{D}$ and $\mathbf{E}_{W}$ are introduced as follows:

$$
\left\{\begin{array}{l}
\mathbf{E}_{D}=\frac{1}{2}\left\|\varepsilon\left(\mathbf{W}^{K}+\mathbf{Z}\left(\mathbf{W}^{K+1}-\mathbf{W}^{K}\right)\right)\right\|^{2}+\lambda\left\|\mathbf{W}^{K+1}-\mathbf{W}^{K}\right\|^{2}, \\
\mathbf{E}_{W}=\frac{1}{N} \sum_{j=1}^{N} \mathbf{w}_{j}^{2},
\end{array}\right.
$$

where $\mathbf{W}$ is the vector of weight and threshold value for network layers; $\mathbf{w}_{j}$, the network weights; $\varepsilon$, the expected error function of output response; $\mathbf{Z}$, the Jacobian matrix of $\varepsilon ; K$, the iteration number; $\lambda$, the iteration variable.

2.3. IESMF Modeling. Since the search accuracy and efficiency of the initial thresholds and weights directly affect the prediction results of ANN-ERSM, we should pay more attention to the search algorithms of the initial weights and thresholds.

Particle Swarm Optimization (PSO) is a kind of random search algorithm based on group collaboration developed through the research on the predation behaviour of biological groups. Because of its fast convergence speed and high search accuracy, some researchers in different fields pay attention to it [30-33]. However, the traditional PSO algorithm using fixed inertial weights is easy to fall into blind search and obtain local optimal solutions, which will greatly affect the search accuracy and efficiency of the global optimal initial weights and thresholds.

To solve the above problems, based on ANN-ERSM and the improved PSO algorithm, an intelligent extreme value agent modeling framework (IESMF) is developed. Give full play to the advantages of the improved PSO algorithm and use it to search for the initial optimal weights and thresholds of IESMF to avoid premature convergence. 


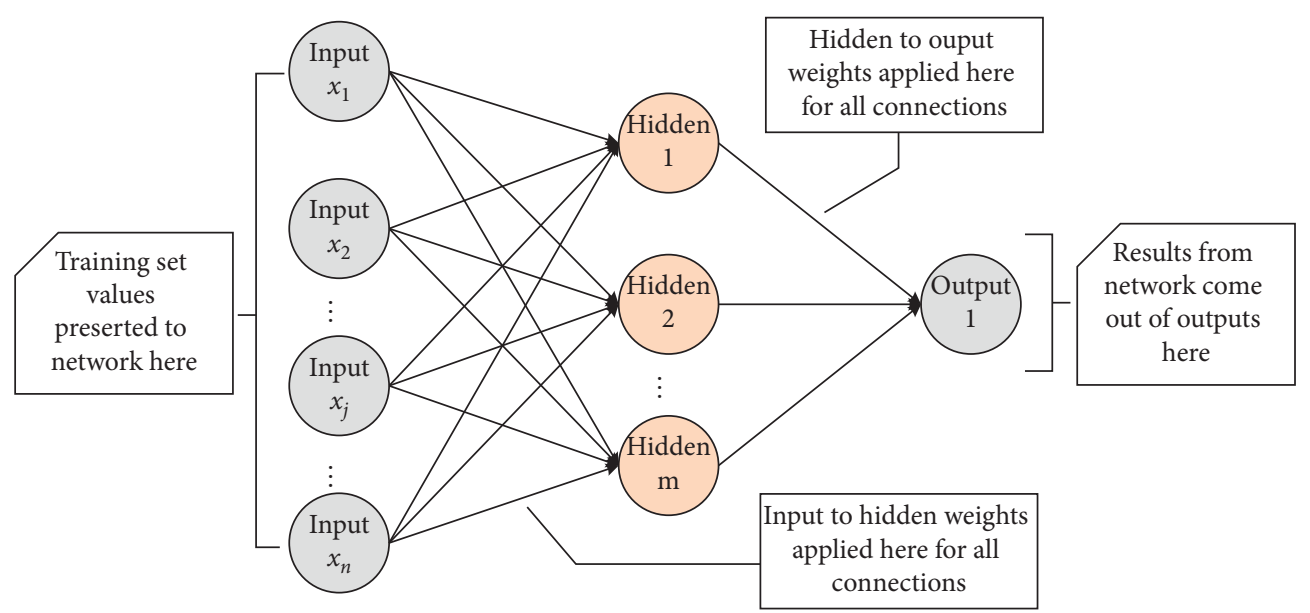

Figure 2: BP-ANN topology model.

The basic idea of the improved PSO algorithm is to use the training error of the BP-ANN model as the fitness value, the weights, and thresholds as the particle position. The process of searching the initial optimal thresholds and weights of the neural network by improved PSO is as follows:

(1) Firstly, initialize a group of particles in space and use the ANN weight and threshold string as the position of the particle. Currently, each particle is a potential solution; (2) the particles are searched according to the current best particles and use the training error function as the fitness function; update the individual position by tracking the individual and group extreme values; (3) update the individual and group extreme value position by selecting the optimal particle until the optimal solution is found, that is, the initial optimal threshold and weight of the neural network.

The update of particle velocity and position is determined by the following:

$$
\left\{\begin{array}{l}
\mathbf{V}_{\mathrm{id}}^{k+1}=\mathbf{w} \mathbf{V}_{\mathrm{id}}^{k}+c_{1} r_{1}\left(\mathbf{P}_{\mathrm{id}}^{k}-\mathbf{X}_{\mathrm{id}}^{k}\right)+c_{2} r_{2}\left(\mathbf{P}_{\mathrm{gd}}^{k}-\mathbf{X}_{\mathrm{gd}}^{k}\right), \\
\mathbf{X}_{\mathrm{id}}^{k+1}=\mathbf{X}_{\mathrm{id}}^{k}+\mathbf{V}_{\mathrm{id}}^{k+1}
\end{array}\right.
$$

where $d$ is the dimension of search space; $\mathbf{w}$, the inertia weight; $i$, the $i$ th particle; $k$, the current iteration number; $\mathbf{V}_{i d}$, the current particle velocity; $\mathbf{X}_{i d}$, the current particle position; $\mathbf{P}_{g d}$, the current population extremum; $\mathbf{P}_{i d}$, the current individual extremum; $r_{1}, r_{2}$ the random numbers during the domain $[0,1] ; c_{1}$ and $c_{2}$ the individual and population learning factor respectively. $\mathbf{w}$ is the inertia weight, representing the extent to which the current velocity of the particle inherits the previous velocity. When the inertial weight is relatively small, the local search ability is strong. On the other hand, the relatively large inertial weight has more advantages in the global search.

Adaptive inertia weight varying with the number of iterations:

$$
\mathbf{w}(t)=w_{1}-\left(w_{1}-w_{2}\right) * \frac{\mathbf{t}}{\mathbf{T}}
$$

where $\mathbf{T}$ is the maximum iteration number; $\mathbf{t}$, the current iteration number; $w_{1}$, the initial inertia weight; $w_{2}$, the inertia weight when iterating to the maximum number of iterations.

The IESMF function is donated by the following:

$$
f(\mathbf{x})=\left\{Y_{j, \max }\left(\mathbf{x}_{j}\right)=f(\mathbf{x}, \mathbf{W}, \mathbf{P}), \mathbf{W} \in \Lambda \mid f: \mathbf{R}^{n} \longrightarrow \mathbf{R}\right\},
$$

where $\mathbf{P}$ is the particle position and velocity of IESMF, $\mathbf{W}$ is the vector of weights and thresholds.

Combining equation (12) with the target allowable value, the limit state function of the complex mechanism is donated by the following:

$$
h_{\mathrm{IESMF}}(\mathbf{x})=y_{\text {allow }}-y_{\mathrm{IESMF}}(\mathbf{x}) .
$$

According to the above analysis, IESMF, which combined with ERSM, ANN, and improved PSO algorithms, is expected to improve the efficiency and accuracy of dynamic probability analysis of complex mechanisms.

The main reasons are as follows: (1) ERSM does not calculate the overall output response, but only focuses on the maximum output response in the analysis time domain, thereby reducing the amount of calculation for each analysis and significantly improving the calculation efficiency; (2) ANN is good at handing nonlinear relationships, so as to improve the calculation accuracy; (3) ANN-ERSM has the advantage of fitting a function through a small number of samples, avoiding a large amount of calculation for solving complex dynamic equations, thereby improving the efficiency of calculation; (4) the improved PSO algorithm with dynamic inertial weight introduced can improve the search accuracy of weights and thresholds in IESMF.

\section{Basic Thought of Probabilistic Analysis}

3.1. Reliability Analysis. Dynamic reliability of the mechanism refers to the ability of the mechanism to perform the predetermined function during movement. The reliability of 
the parameters such as displacement, velocity, and stress in the specified range is included [12].

It is assumed that the mechanism system consists of $n$ components, $\mathbf{S}_{i}\left(\mathbf{x}_{i}, t\right)$, is the dynamic response of component $i(i=1,2, \ldots, n)$ in the motion time domain $[0, \mathrm{~T}]$, which is a complex random process, where $\mathbf{x}_{i}$ is the random variable corresponding to the dynamic response of component $i . \mathbf{S}_{i}\left(\mathbf{x}_{i}, \mathrm{t}\right)$ is the dynamic response of component $i$ at time $t$.

In the motion time domain $[0, T], \mathbf{D}_{i}\left(\mathbf{x}_{i}, \mathrm{t}\right)$ is the output response of component $i$ at time $t$, and $\left[\mathbf{D}_{\boldsymbol{i}}\right]$ is the allowable value. The reliability of component $i$ can be expressed as follows:

$$
\mathbf{R}_{i}(\mathbf{t})=\mathbf{P}\left\{\mathbf{D}_{i}\left(\mathbf{x}_{i}, \mathbf{t}\right) \leq\left[\mathbf{D}_{i}\right]\right\} .
$$

The output extremum response of component $i$ in the motion time domain $[0, \mathrm{~T}]$ is $\mathbf{S}_{i}\left(\mathbf{x}_{i}, \mathbf{t}\right)$, and the allowable value is $\left[S_{i}\right]$, then the reliability of component $i$ can be expressed as follows:

$$
\mathbf{R}_{i}=\mathbf{P}\left\{\mathbf{S}_{i}\left(\mathbf{x}_{i}\right) \leq\left[\mathbf{S}_{i}\right]\right\} .
$$

3.2. Essential Process. Reliability analysis is to use the random variable analysis mechanism with random dispersion characteristics to meet the probability of the specified function under the specified conditions within the specified time in the actual project. Combining ERSM, ANN, and improved PSO algorithm, the IESMF method for reliability analysis of complex mechanism is established. Figure 3 shows the flow of dynamic reliability analysis of the complex mechanism using IESMF.

As shown in Figure 3, the basic idea of using IESMF for dynamic reliability analysis of complex mechanism is described as follows:

(1) First, according to the characteristics of complex mechanism, combine the comprehensive modal theory with mechanical system dynamics theory, determine the random input variables, and establish the transient dynamics finite element model of the mechanism

(2) According to the finite element model, combined with the deterministic analysis results, extract a sufficient number of samples as training samples

(3) To make the neural network model suitable for these training samples, the structure of the neural network is defined by referring to the characteristics of input random variables and the output response, such as the number of nodes in each layer

(4) Use the BR algorithm to establish a neural network extreme value response surface model

(5) Employ the improved PSO to search the initial optimal weight and threshold of IESMF; finally, use the Monte Carlo method to sample the IESMF to obtain the output response value

\section{Case Study}

The rack-and-pinion steering mechanism (RPSM) of the nose landing gear is a typical complex mechanism with timevarying, high-nonlinearity, and multiparameters characteristics, which is applied to many existing aircraft [34]. In this study, a certain type of nose landing gear RPSM is the research object. Considering the dynamic operation process of speed, load, and relative position with time, the dynamic probability analysis of RPSM will be carried out in this section to verify the proposed IESMF.

4.1. Problem Description. The landing gear system determines the safe take-off and landing performance of the aircraft and is the key load-bearing part in the take-off and landing process. Studies have shown that up to $40 \%$ of aircraft accidents are caused by landing gear failures, which can lead to extremely serious consequences [34]. Among them, the performance of the nose wheel steering system is a factor that cannot be ignored. To improve the safety and flexibility of aircraft ground control, the nose wheel steering system has been implemented in the full range of aircraft ground speeds. Frequent ground handling and complex working environment put forward higher requirements on the fatigue performance and functional reliability of the front wheel turning mechanism.

The power source of the front wheel turning mechanism is a hydraulic drive system, which controls the flow of hydraulic oil to the left/right cavity of the turning control actuator by adjusting the hydraulic reversing valve, which determines the movement of the hydraulic piston connected to the steering shaft (Figure 4). The pinion is linked to the steering axis, while the rack is inserted between the two strokes [2]. Pistons located at both ends of the rack push the rack to reciprocate linearly. The linear motion of the rack is transformed into the rotation of the gear through the gear and rack meshing transmission to drive the steering axis to rotate, as shown in Figure 5. The RPSM is easy to achieve a large operating angle and has a compact structure. The meshing motion of the rack-and-pinion is an important part of the turning mechanism to realize the turning motion, which has a great impact on the load-bearing capacity and reliability of the turning mechanism.

4.2. Deterministic Analysis. The probabilistic design of the RPSM of the nose landing gear is based on the failure mode of the gear root stress. To realize the dynamic probability analysis of RPSM, the first work is to establish the threedimensional model and FE model of the research object. The three-dimensional model and the finite element model of RPSM are obtained after reasonable simplification of the real rack-and-pinion steering mechanism of a nose landing gear which is shown in Figures 6 and 7.

Choosing a reasonable mesh type and size can improve the accuracy and efficiency of finite element analysis $[35,36]$. To ensure the accuracy of the calculation, the meshing 


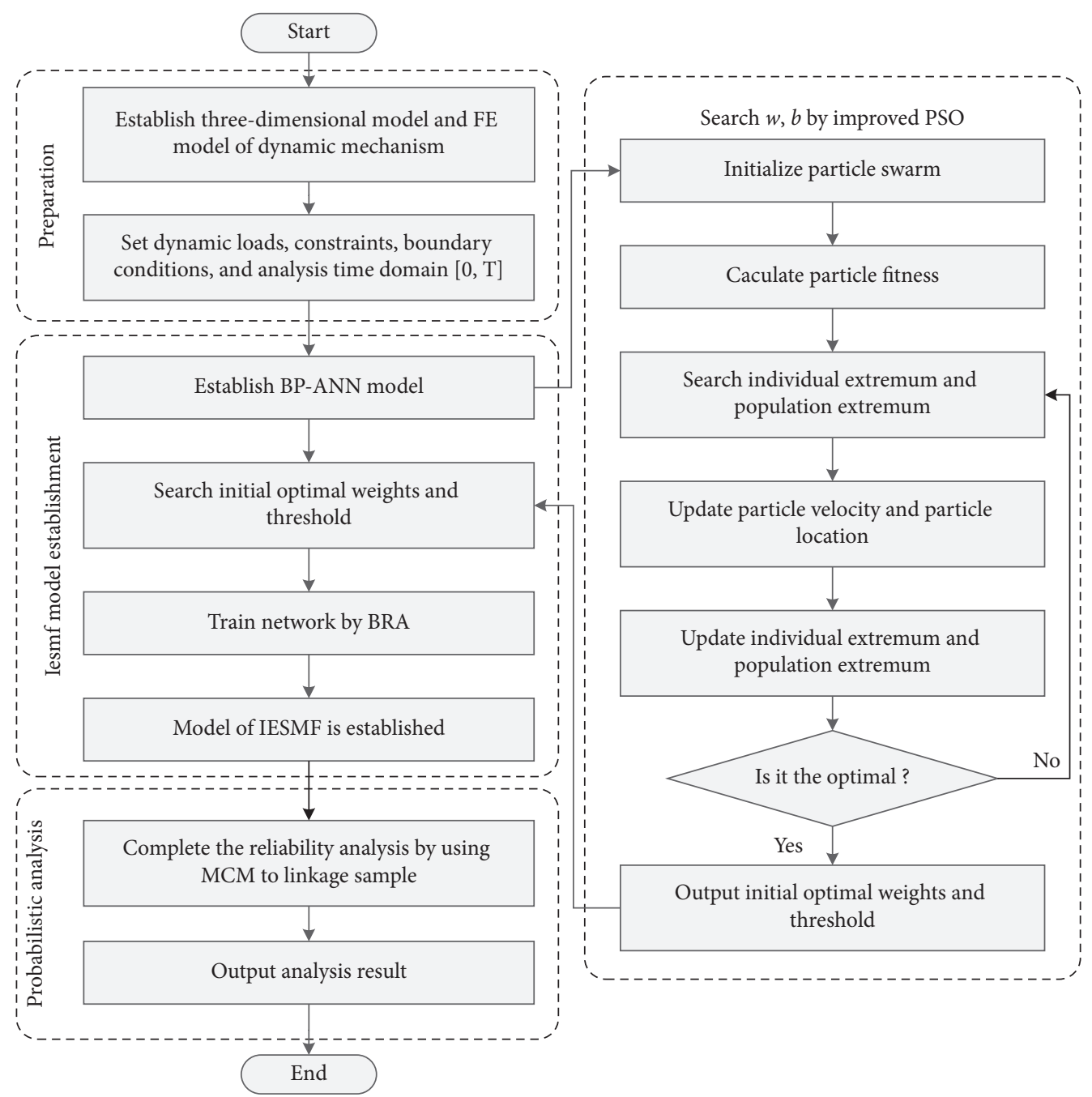

FIGURE 3: Essential process of probabilistic analysis for dynamic reliability with IESMF.

position between the tooth root and the tooth is small, and the larger mesh is used for the rest of the structure to reduce the calculation time. As shown in Figure 7, the FE model of rack-and-pinion is composed of tetrahedral elements, including 382133 nodes and 263567 nodes, 109539 nodes and 72294 elements, respectively.

$300 \mathrm{M}$ steel is used as the material of the rack-andpinion. Table 1 shows its mechanical properties. In the turning process, the motion cycle is 11 s. Figure 8 shows the change curve of the gear speed in the analysis time domain [0s, 11s] in a cycle.

The dynamic finite element simulation is carried out in ANSYS, and a series of stress cloud diagrams during the gear transmission process are obtained, as shown in Figure 9. According to the finite element simulation calculation, the meshing process, and characteristics of the RPSM can be obtained, and the root stress of the gear has a certain periodicity. Figure 10 is the meshing stress cloud diagram of the RPSM. The analysis shows that when the double-tooth meshing of RPSM is transformed into single-tooth meshing, the gear root stress reaches the maximum. This is because when the double-tooth mesh is converted to single-tooth, the contact part is located at the highest point of single-tooth mesh, so the root stress of gear reaches the maximum.

\subsection{Dynamic Probabilistic Analysis of RPSM}

4.3.1. Establish IESMF Mathematical Model. Based on the dynamic deterministic analysis of RPSM, considering the operating conditions and uncertainty of material parameters, the material density $\rho$, friction coefficient $\mu$, turning rate $v$, and load $M$ are selected as the random parameters of RPSM, which are shown in Table 2. In the probabilistic analysis, we assume that these random variables are independent of each other and obey a normal distribution.

According to the distribution characteristics of input random variables in Table 2, 50 groups of input data are extracted by the Latin hypercube sampling (LHS) method. Through 50 times of dynamic deterministic analysis, the maximum root stress corresponding to the input data was obtained to form 50 samples. Among them, 30 are used as training samples to create IESMF, and 20 are used as test samples for IESMF verification. 


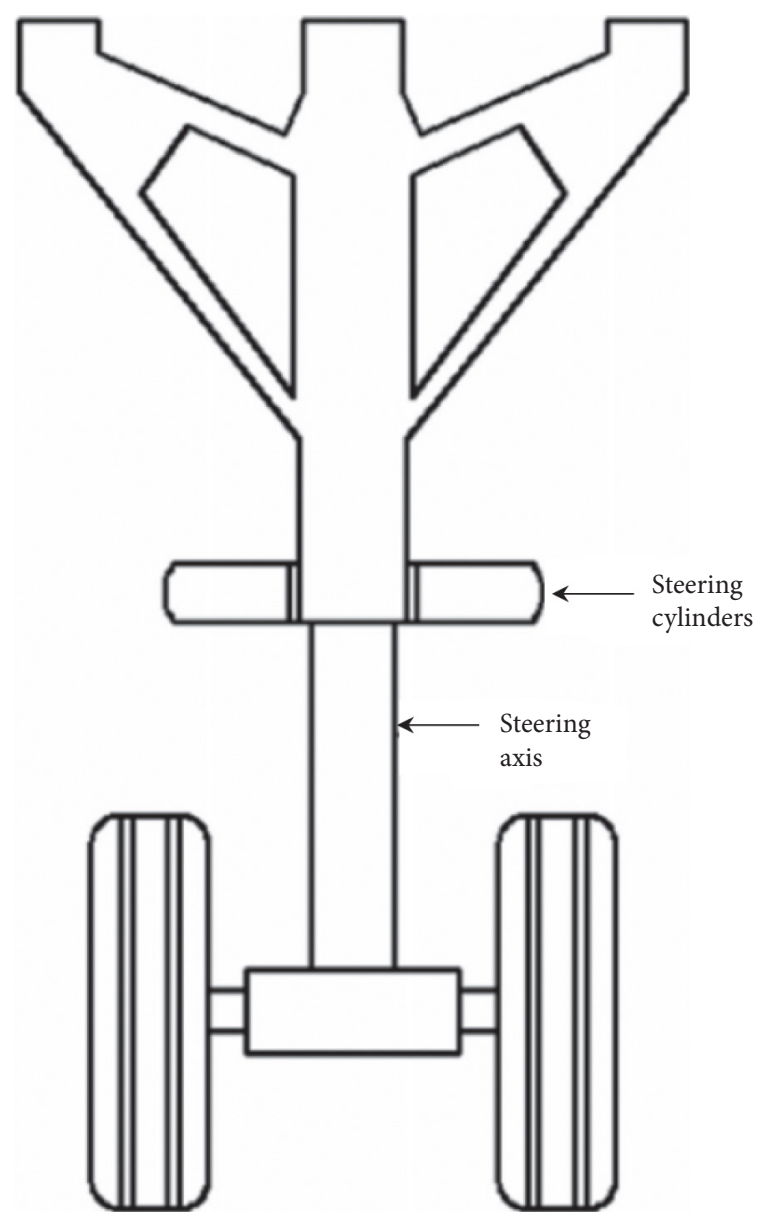

Figure 4: Nose landing gear with steering cylinders.

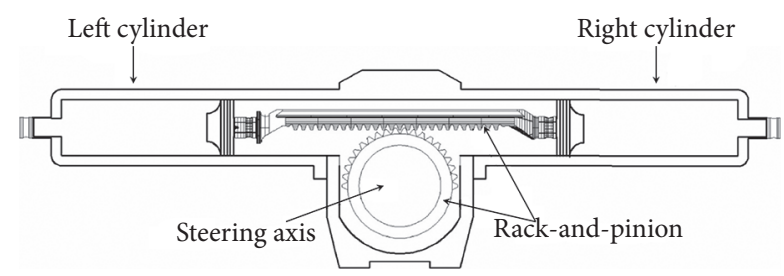

FIGURE 5: Rack-and-pinion steering mechanism (RPSM) of a nose landing gear.
To make IESMF suitable for these training samples, according to the characteristics of input random variables and output response, the ANN model uses a 4-3-1 threelayer structure. Choose 'tansig' as the transfer function from the input layer to the hidden layer; 'purelin' as the transfer function from the hidden layer to the output layer; 'trainbr' as the training function. The number of particles $N=40$ and the particle dimension $\theta=16$ are selected for the model. After 100 iterations, Figure 11 shows the optimal fitness value curves.

The initial optimal weights and threshold values are inputted into the ANN model. Using the Bayesian regularization (BR) algorithm for network training, the IESMF function can be obtained, where the weights and thresholds are shown in the following equation:

$$
\left\{\begin{array}{l}
\mathbf{w}_{1}=\left[\begin{array}{cccc}
0.2749 & 0.6536 & -0.3338 & 0.3520 \\
-0.1257 & -0.0009 & -0.6495 & 0.1037 \\
0.0699 & -0.4073 & -0.5431 & -0.2979
\end{array}\right], \\
\mathbf{b}_{1}=\left[\begin{array}{l}
-0.7589 \\
-2.7129 \\
-3.0362
\end{array}\right], \\
\mathbf{w}_{2}=\left[\begin{array}{lll}
-0.5973 & -0.8071 & -0.7876
\end{array}\right], \\
\mathbf{b}_{2}=[-0.0228],
\end{array}\right.
$$

Combining 20 test samples, the IESMF modeling characteristic analysis was carried out by comparing the predicted value with the true value, and the result is shown in Figure 12. The predicted value of IESMF is basically the same as the real value obtained by deterministic analysis, and its prediction error is within the acceptable range of the project. Therefore, IESMF can meet the prediction accuracy requirements.

4.3.2. Reliability Analysis of RPSM. Through 10000 simulations of IESMF through MC simulation, the output responses are obtained by inversed normalization. The simulation history and distribution histogram of gear root stress are shown in Figure 13. The number of response values greater than the set allowable value is defined as the number of failures. The ratio of the number of response values less than the set allowable value to the total number of responses is defined as reliability. The gear root stress obeys the normal distribution with the mean value of $780.12 \mathrm{MPa}$ and the standard deviation of $25.42 \mathrm{MPa}$. When the allowable value is $850 \mathrm{MPa}$, the failure number is 29 and the reliability is 0.9971 . The allowable value is determined by the $3 \sigma$ principle based on the mean value and standard deviation of the gear root stress.

4.4. Validation of IESMF. To verify the applicability and performance of the IESMF proposed in this research, the reliability analysis of RPSM of nose landing gear is carried out using IESMF, MC simulation, and ERSM, respectively. The calculation time and accuracy are shown in Table 3.

FIgURE 6: Three-dimensional RPSM. 

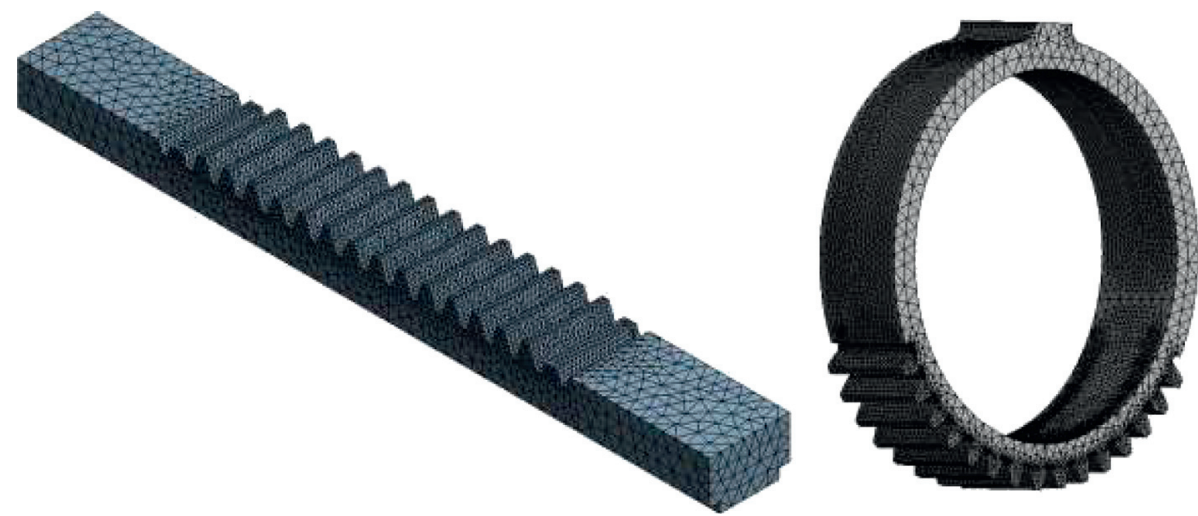

FIgURE 7: Finite element (FE) model of the gear and rack.

TABLe 1: Mechanical property parameters of $300 \mathrm{M}$ steel.

\begin{tabular}{lccccc}
\hline Material parameters & $\sigma_{\mathrm{b}}(\mathrm{MPa})$ & $\sigma_{0.2}(\mathrm{MPa})$ & $\delta(\%)$ & $\varphi(\%)$ & $E(\mathrm{GPa})$ \\
\hline Value & 1963 & 1615 & 11.3 & 46.9 & 199 \\
\hline
\end{tabular}

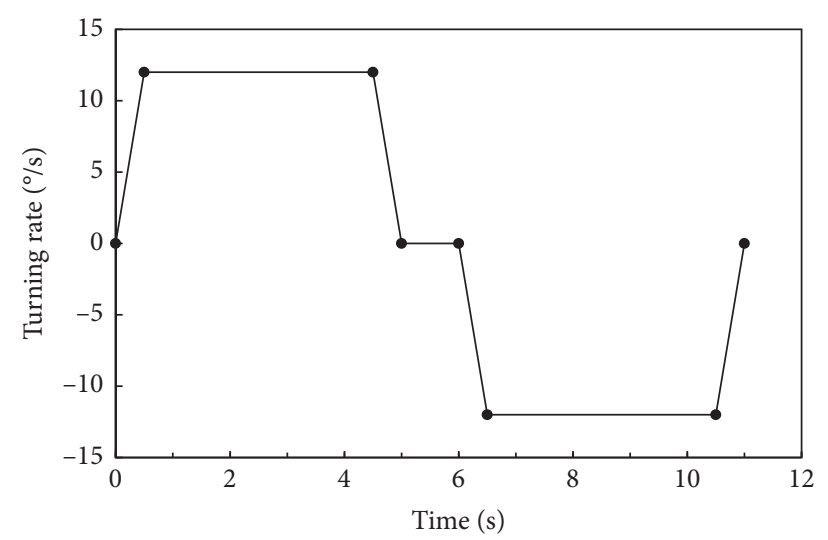

FIgURE 8: Angular velocity curve of the steering axis.
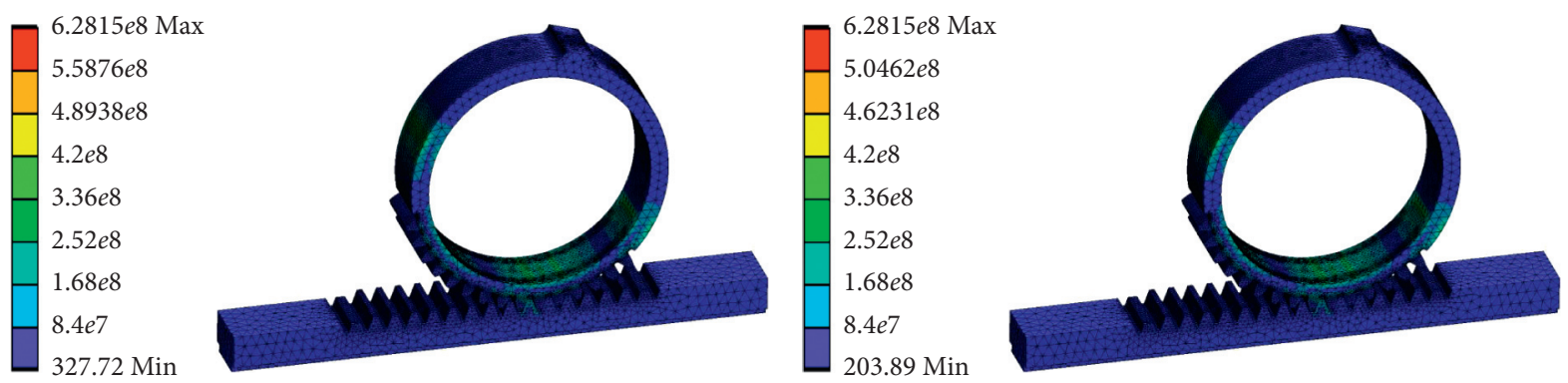

(a)

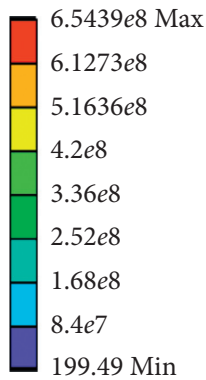

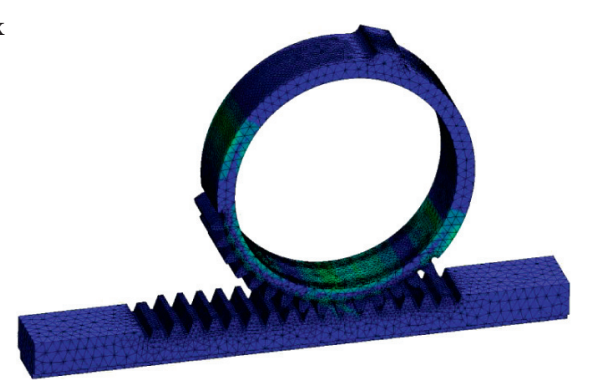

(c) (b)
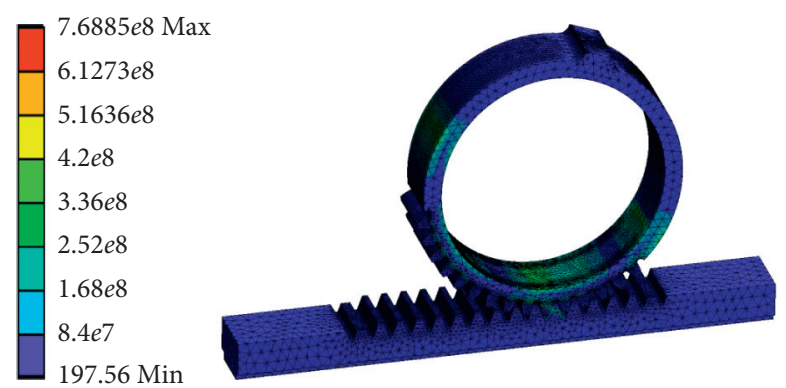

(d)

FIgURE 9: Stress nephogram during gear rotation. 


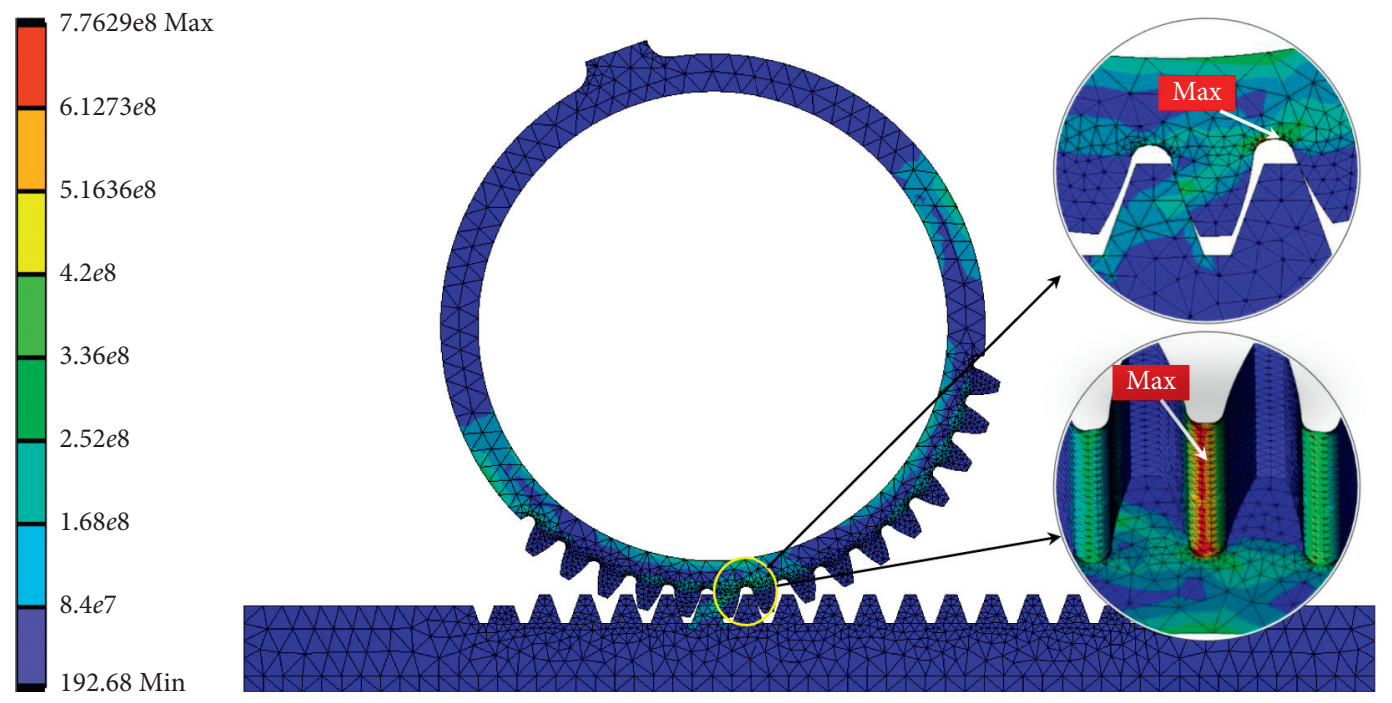

Figure 10: The maximum stress position of the gear root.

TABLE 2: Distribution features of input random variables.

\begin{tabular}{lcccc}
\hline Random variable & $\rho\left(\mathrm{kg} \cdot \mathrm{m}^{-3}\right)$ & $\mu$ & $V(\% / \mathrm{s})$ & $M_{f}(\mathrm{~N} \cdot \mathrm{m})$ \\
\hline Mean & 7740 & 0.05 & 12 & 1910 \\
Standard deviation & 232 & 0.00333 & 1.33 & 66.67
\end{tabular}

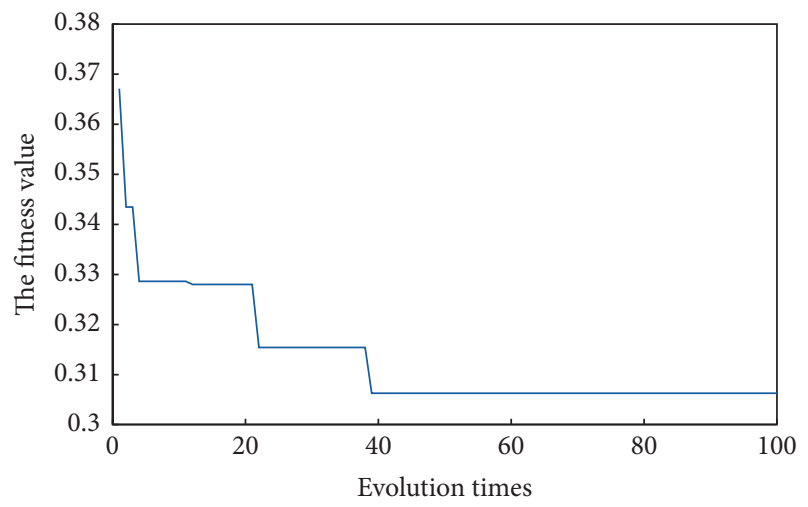

FIgUre 11: Optimal fitness value curves.

As shown in Table 3, IESMF and ERSM are far less than MC in terms of calculation time. As the number of simulations increases, the computational efficiency of IESMF is significantly higher than that of ERSM. The results show that IESMF, which combines ANN and PSO based on the ERSM idea, has a higher computational efficiency in the analysis of mechanism dynamic reliability. The reason is that: (i) Thanks to the fact that ERSM only pays attention to the extreme value of the dynamic response, the nonlinear response surface function can be quickly fitted. (ii) ANN has fast and accurate characteristics in training for uncertain parameters. (iii) Using the improved PSO algorithm to optimize the initial weights and thresholds, IESMF can be obtained quickly, and the computational efficiency of dynamic reliability analysis of complex mechanisms is improved.

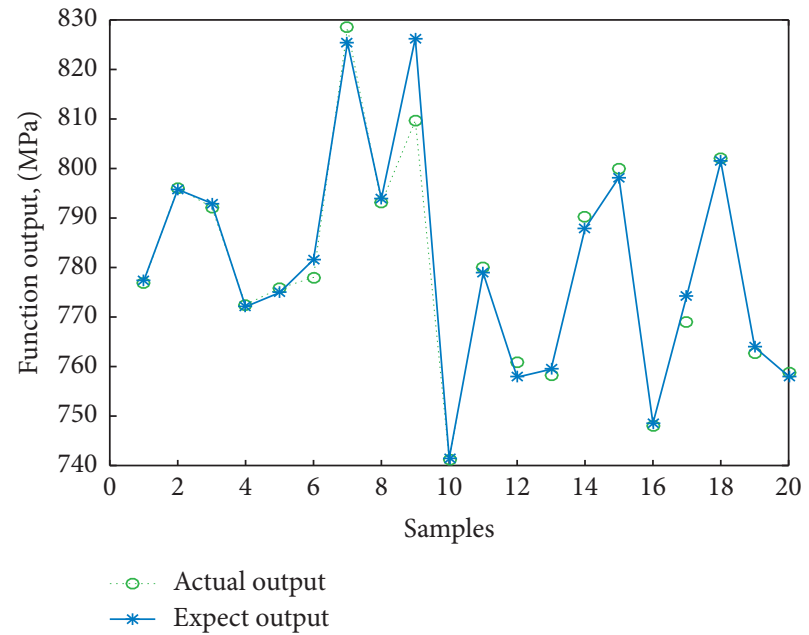

FIgURE 12: Prediction results of IESMF.

Regarding the calculation accuracy of IESMF, almost all sampling points are fitted by it with low training error, showing the strong generalization ability and nonlinear fitting ability of IESMF. As shown in Table 3, compared with ERSM, IESMF is more precise and similar to the MC method. The reasons are as follows: (i) IESMF combined with BP-ANN has excellent generalization ability and nonlinear mapping ability. The mathematical model is more accurate, and the calculation accuracy is higher. (ii) The BR algorithm reduces the training error by reducing the weight of the network. (iii) Combined with the improved PSO algorithm, the search accuracy of global initial optimal weights and thresholds is improved.

To sum up, IESMF guarantees the calculation accuracy and greatly improves the calculation efficiency. This method has good adaptability to dynamic probability analysis of complex mechanisms, and its comprehensive performance is satisfactory. 


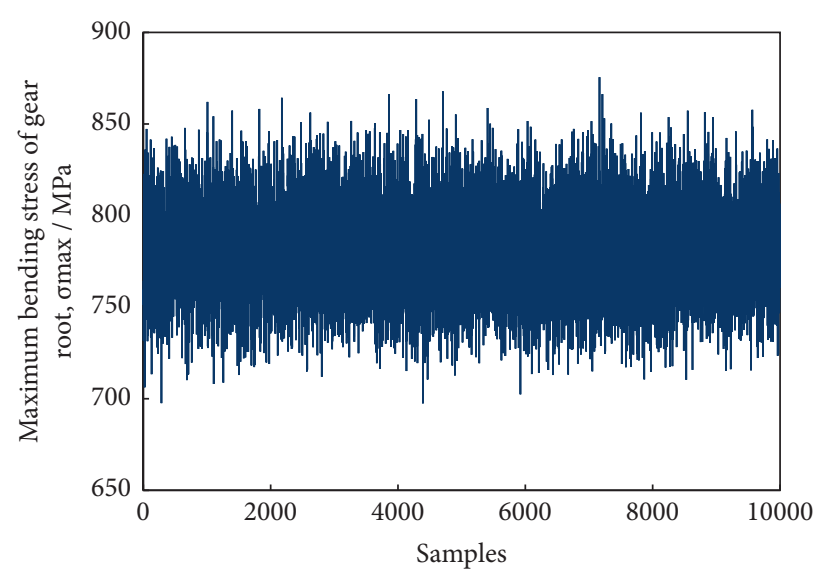

(a)

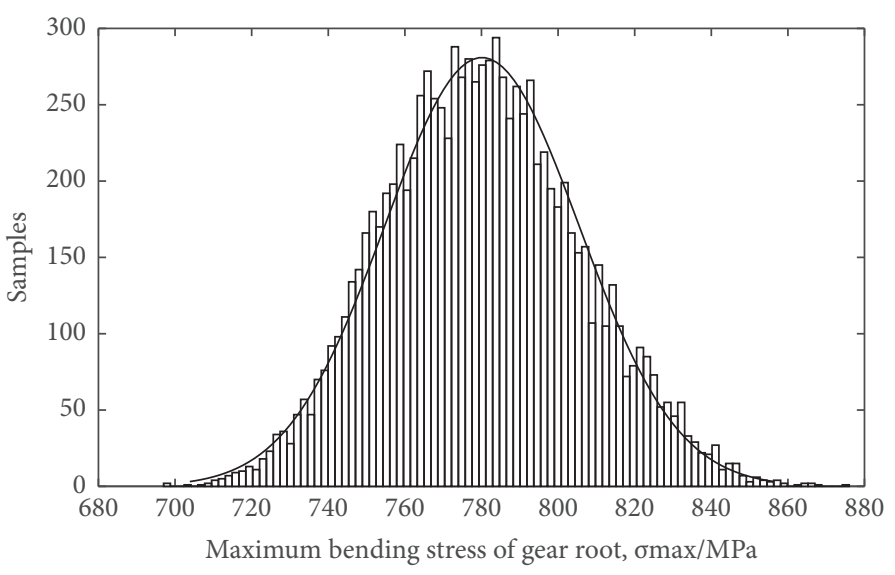

(b)

FiguRE 13: Simulation history and distribution histogram of gear root stress. (a) Simulation history of gear root stress. (b) Distributions of gear root stress output response.

TABLE 3: Reliability degree of dynamic probabilistic analysis on RPSM.

\begin{tabular}{|c|c|c|c|c|c|c|c|c|}
\hline \multirow{2}{*}{ Simulation } & \multicolumn{3}{|c|}{ Simulation time, $\mathrm{s}$} & \multicolumn{3}{|c|}{ Reliability } & \multicolumn{2}{|c|}{ Precision, \% } \\
\hline & $\mathrm{MC}$ & ERSM & IESMF & MC & ERSM & IESMF & ERSM & IESMF \\
\hline $10^{2}$ & $6.34 \times 10^{3}$ & 0.4693 & 0.2489 & 0.99 & 0.98 & 0.99 & 98.92 & 100 \\
\hline $10^{3}$ & $7.48 \times 10^{4}$ & 2.7359 & 0.8357 & 0.997 & 0.992 & 0.998 & 99.03 & 99.9 \\
\hline $10^{4}$ & - & 6.7876 & 2.0153 & - & 0.9934 & 0.9971 & 98.97 & 99.99 \\
\hline
\end{tabular}

\section{Conclusions}

The purpose of this research is to establish an efficient and high-precision surrogate model, called the intelligent extremum surrogate modeling framework (IESMF), which is used to process the probabilistic analysis of complex mechanisms with time-varying, high-nonlinearity, and multiparameters characteristics. The feasibility and effectiveness of the method are verified by analysing and calculating the engineering examples of rack-and-pinion steering mechanism (RPSM). Some conclusions are summarized as follows.

(1) The distribution features and simulation history of output responses under the gear root stress failure mode of RPSM are obtained. RPSM stress reliability probability is 0.9971 .

(2) The improved PSO algorithm has an excellent performance in finding the optimal initial weight and threshold of the network. The IESMF established by combining ERSM, ANN, and improved PSO can successfully complete the dynamic reliability analysis of complex mechanisms.

(3) By comparing with traditional methods, the results show that the IESMF proposed in this research has high efficiency and high precision for dynamic reliability analysis of complex mechanisms. As the number of simulations increases, the advantages of IESMF are more obvious.

(4) The work in this paper enriches the theory of probabilistic analysis and provides a promising approach for the optimal design of complex mechanisms in the fields of aerospace engineering and mechanical engineering.

\section{Data Availability}

The data used to support the findings of this study are included within the article.

\section{Conflicts of Interest}

The authors declare that there are no conflicts of interest regarding the publication of this paper.

\section{Acknowledgments}

This paper was supported by the National Natural Science Foundation of China (Grant no. 51875465). The authors would like to thank them.

\section{References}

[1] S. Pragadheswaran and P. K. Balguri, "Conceptual design and linear static analysis of nose landing gear," in Proceedings of the International Conference On Robotics, Automation, Control And Embedded Systems (RACE), Hindustan University, Chennai, India, February 2015.

[2] L. P. Di Noia, R. Rizzo, and R. Rizzo, "Design of a five-phase permanent-magnet motor for the electric steering of an aircraft nose landing gear," IET Electrical Systems in Transportation, vol. 7, no. 4, pp. 327-333, 2017. 
[3] S. P. Zhu, B. Keshtegar, S. Chakraborty, N. T. Trung. Novel probabilistic model for searching most probable point in structural reliability analysis. Computer Methods in Applied Mechanics and Engineering, 2020, vol. 366, Article ID. 113027.

[4] A. A. Razi-Kazemi, K. Niayesh, and R. Nilchi, "A probabilistic model-aided failure prediction approach for spring-type operating mechanism of high-voltage circuit breakers," IEEE Transactions on Power Delivery, vol. 34, no. 4, pp. 1280-1290, 2019.

[5] S. P. Zhu, B. Keshtegar, M. Bagheri, P. Hao, N. T. Trung. Novel hybrid robust method for uncertain reliability analysis using finite conjugate map. Computer Methods in Applied Mechanics and Engineering, 2020, vol. 371, Article ID. 113309.

[6] K. W. Chase, J. Gao, and S. P. Magleby, "General 2-D tolerance analysis of mechanical assemblies with small kinematic adjustments," Journal of Design and Manufacturing, vol. 5, no. 4, pp. 263-274, 1995.

[7] Y. Yin, K. Xu, and H. Nie, "Dynamics analysis of spatial landing-gear mechanism with hinge clearance and Axis deviation," Journal of Aircraft, vol. 58, pp. 1-13, 2020.

[8] E. Zheng, R. Zhu, S. Zhu, and X. Lu, "A study on dynamics of flexible multi-link mechanism including joints with clearance and lubrication for ultra-precision presses," Nonlinear Dynamics, vol. 83, no. 1-2, pp. 137-159, 2016.

[9] X. Y. Gong, C. J. Xue, and J. B. Xu, "Dynamics simulation study on civil aircraft planned pavement emergency landing," Journal of Vibroengineering, vol. 17, no. 8, pp. 4496-4506, 2015.

[10] C. Lu, Y.-W. Feng, R. P. Liem, and C.-W. Fei, "Improved Kriging with extremum response surface method for structural dynamic reliability and sensitivity analyses," Aerospace Science and Technology, vol. 76, pp. 164-175, 2018.

[11] L.-K. Song, C.-W. Fei, G.-C. Bai, and L.-C. Yu, "Dynamic neural network method-based improved PSO and BR algorithms for transient probabilistic analysis of flexible mechanism," Advanced Engineering Informatics, vol. 33, pp. 144-153, 2017.

[12] L.-K. Song, J. Wen, C.-W. Fei, and G.-C. Bai, "Distributed collaborative probabilistic design of multi-failure structure with fluid-structure interaction using fuzzy neural network of regression," Mechanical Systems and Signal Processing, vol. 104, pp. 72-86, 2018.

[13] Q. Lv, "Probabilistic analysis of underground shock excavations using response surface method and SORM," Computers and Geotechnics, vol. 38, no. 8, pp. 1008-1021, 2011.

[14] S. D. Lawley, "A probabilistic analysis of volume transmission in the brain," SIAM Journal on Applied Mathematics, vol. 78, no. 2, pp. 942-962, 2018.

[15] K. Jenkins, J. Hall, V. Glenis, and C. Kilsby, "A probabilistic analysis of surface water flood risk in london," Risk Analysis, vol. 38, no. 6, pp. 1169-1182, 2018.

[16] S. K. Au, "Probabilistic failure analysis by importance sampling Markov chain simulation," Journal of Engineering Mechanics, vol. 130, no. 3, pp. 303-311, 2004.

[17] Y. Wang, Z. Cao, and S.-K. Au, "Efficient Monte Carlo simulation of parameter sensitivity in probabilistic slope stability analysis," Computers and Geotechnics Comp, vol. 37, no. 7-8, pp. 1015-1022, 2010.

[18] Y. M. Asri, E. A. Azrulhisham, A. W. Dzuraidah, A. Shahrir, A. Shahrum, and Z. Azami, "Fatigue life reliability prediction of a stub axle using Monte Carlo simulation," International
Journal of Automotive Technology, vol. 12, no. 5, pp. 713-719, 2011.

[19] T. Cho, M.-K. Song, and D. H. Lee, "Reliability analysis for the uncertainties in vehicle and high-speed railway bridge system based on an improved response surface method for nonlinear limit states," Nonlinear Dynamics, vol. 59, no. 1-2, pp. 1-17, 2010.

[20] S. E. Fang, Q. H. Zhang, and W. X. Ren, "Parameter variability estimation using stochastic response surface model updating," Mechanical Systems and Signal Processing, vol. 49, no. 1-2, pp. 249-263, 2014.

[21] Y.-S. Eom, K.-S. Yoo, J.-Y. Park, and S.-Y. Han, "Reliabilitybased topology optimization using a standard response surface method for three-dimensional structures," Structural and Multidisciplinary Optimization, vol. 43, no. 2, pp. 287-295, 2011.

[22] C. Zhang, L. Song, C. Fei, C. Lu, and Y. Xie, "Advanced multiple response surface method of sensitivity analysis for turbine blisk reliability with multi-physics coupling," Chinese Journal of Aeronautics, vol. 29, no. 4, pp. 962-971, 2016.

[23] C.-W. Fei, W.-Z. Tang, and G.-C. Bai, "Novel method and model for dynamic reliability optimal design of turbine blade deformation," Aerospace Science and Technology, vol. 39, no. 6, pp. 588-595, 2014.

[24] C.-W. Fei, Y.-S. Choy, D.-Y. Hu, G.-C. Bai, and W.-Z. Tang, "Transient probabilistic analysis for turbine blade-tip radial clearance with multi-component and multi-physics fields based on DCERSM," Aerospace Science and Technology, vol. 50, no. 3, pp. 62-70, 2016.

[25] A. H. A. AL-Waeli, K. Sopian, H. A. Kazem et al., "Comparison of prediction methods of PV/T nanofluid and nanoPCM system using a measured dataset and artificial neural network," Solar Energy, vol. 162, pp. 378-396, 2018.

[26] H. G. Zhang, Z. S. Wang, and D. R. Liu, "A comprehensive review of stability analysis of continuous-time recurrent neural networks," IEEE Transactions on Neural Networks and Learning Systems, vol. 7, no. 25, pp. 1229-1262, 2014.

[27] L. Liu, Z. S. Wang, and H. W. Zhang, "Adaptive fault-tolerant tracking control for MIMO discrete-time systems via reinforcement learning algorithm with less learning parameters," IEEE Transactions on Automation Science and Engineering, vol. 14, no. 1, pp. 1-15, 2017.

[28] D. Carvalho, N. A. Garcia-Martinez, J. L. Lado, and J. Fernandez-Rossier, "Real-space mapping of topological invariants using artificial neural networks," Physical Review B, vol. 97, no. 11, pp. 115453.1-115453.8, 2018.

[29] A. H. Ala, "Adaptive artificial neural network-based models for instantaneous power estimation enhancement in electric vehicles' Li-ion batteries," IEEE Transactions on Industry Applications, vol. 55, no. 1, pp. 840-849, 2019.

[30] J. Kennedy, "Particle swarm optimization," in Proceedings of the 1995 IEEE International. Conference on. Neural Networks, vol. 4, no. 8, pp. 1942-1948, Perth, Australia, December 2011.

[31] S. Sun and J. Li, "Parameter estimation of methanol transformation into olefins through improved particle swarm optimization with attenuation function," Chemical Engineering Research and Design, vol. 92, no. 11, pp. 2083-2094, 2014.

[32] J. Matos, R. P. V. Faria, I. B. R. Nogueira, J. M. Loureiro, and A. M. Ribeiro, "Optimization strategies for chiral separation by true moving bed chromatography using Particles Swarm Optimization (PSO) and new Parallel PSO variant," Computers \& Chemical Engineering, vol. 123, no. 6, pp. 344-356, 2019. 
[33] A. Sedki and D. Ouazar, "Hybrid particle swarm optimization and differential evolution for optimal design of water distribution systems," Advanced Engineering Informatics, vol. 26, no. 3, pp. 582-591, 2012.

[34] Q.-C. Chang and C.-J. Xue, "Reliability analysis and experimental verification of landing-gear steering mechanism considering environmental temperature," Journal of Aircraft, vol. 55, no. 3, pp. 1154-1164, 2018.

[35] C. Lu, Y.-W. Feng, C.-W. Fei, and S.-Q. Bu, "Improved decomposed-coordinated kriging modeling strategy for dynamic probabilistic analysis of multicomponent structures," IEEE Transactions on Reliability, vol. 69, no. 2, pp. 440-457, 2020.

[36] M. Soltani, R. Kulkarni, T. Scheinost, T. Groezinger, and A. Zimmermann, "A novel approach for reliability investigation of LEDs on molded interconnect devices based on FEanalysis coupled to injection molding simulation," IEEE Access, vol. 7, pp. 56163-56173, 2019. 